\title{
Mineração
}

\section{Avaliação da perda da coloração artificial de ágatas}

\section{(Color loss evaluation of artificially stained agates)}

\author{
Rodrigo de Almeida Silva \\ Mestre em Engenharia, PPGEM/UFRGS \\ E-mail: rodrigoigo@ct.ufrgs.br \\ Carlos Otavio Petter \\ Professor, Dr, DEMIN/PPGEM/UFRGS \\ E-mail:cpetter@ufrgs.br \\ Ivo André H. Schneider \\ Professor, Dr., DEMET/PPGEM/UFRGS \\ E-mail: ivoandre@ct.ufrgs.br
}

\section{Resumo}

O Rio Grande do Sul é um dos principais produtores de geodos de ágatas do mundo. As ágatas da região de Salto do Jacuí permitem a introdução de alguns corantes em seus poros, tornando-as coloridas. O objetivo do presente trabalho foi estudar a perda de cor de ágatas tingidas artificialmente, considerando diferentes ambientes de exposição. Estudaram-se ágatas coloridas pelos procedimentos clássicos (verde, vermelho, azul e preto) e coloridas com corantes orgânicos (verde, vermelho, rosa e roxo). O procedimento experimental consistiu em expor as chapas de ágatas em três ambientes com luminosidades distintas: escuro, doméstico e ao ar livre. A evolução da cor foi avaliada pela técnica de colorimetria diferencial, usando o sistema colorimétrico CIE L*a*b*. Os resultados obtidos demonstram que os métodos clássicos de tingimento proporcionam cores permanentes, enquanto que os corantes orgânicos promovem cores instáveis. Dependendo do grau de luminosidade e tempo de exposição, a perda de cor pode ser praticamente total.

Palavras-chave: Ágatas, tingimento, cor, fotodegradação.

\begin{abstract}
The State of Rio Grande do Sul in Brazil is one of the main world producers of agates. The gray type of agates found in Salto do Jacuí is specially suited to be stained in various colours. The purpose of this work was to evaluate the colour loss of artificially stained agates, considering different exposure environments. Studies were carried out with agates stained by classical procedures (green, red, blue, and black) and with organic dyes (green, red, pink, and purple). The experimental procedure consisted of displaying agate plates in three places with distinct luminosity: dark, indoor/domestic, and outdoor. The color variation was analyzed by differential colorimetry, using the CIE $L * a * b *$ colorimetrical system. The results show that the classical procedures provided permanent colours, while the colour given by organic dyes was unstable. Depending on the luminosity and exposure time, the colour loss can be practically complete.
\end{abstract}

Keywords: Agate, coloring, colour, photodegradation. 


\section{Introdução}

O Brasil é um dos principais exportadores de ágatas em estado bruto para o mundo. Esse bem gemológico é encontrado no Estado do Rio Grande do Sul, principalmente na região do Planalto Médio. Grande parte da produção destina-se para fins ornamentais, onde as gemas são trabalhadas para confecção de objetos de arte e adornos, entre os quais chapas finas e grossas, encosto de livros, cinzeiros, relógios, pirâmides, esferas, ovos, obeliscos, espátulas, artigos para cozinha, entre outros (DNPM, 1998).

As ágatas do Rio Grande do Sul têm, como característica, uma estrutura bandeada (Sampaio e Tubino, 1999). Os grãos de quartzo formam bandas fibrosas, produzindo diferentes graus de porosidade, característica física fundamental para a impregnação dos agentes responsáveis pela coloração artificial.

Atualmente existem várias maneiras para colorir as ágatas. Os métodos clássicos foram desenvolvidos pelos alemães no século XIX e aplicados no Rio Grande do Sul desde o início do século XX. As ágatas são imersas em uma solução que contém íons metálicos ou açúcar, que, após impregnarem os poros da pedra ornamental, são submetidos a uma reação química, com a finalidade de convertê-los em um composto colorido e insolúvel (Knecht, 1957). Recentemente, também têm se realizado o tingimento com corantes orgânicos (anilinas). O processo consiste, basicamente, na imersão das ágatas em soluções alcoólicas de corante por um período suficiente para permitir a penetração das moléculas de corante nos poros das pedras (Pizzolato et al., 2002). Um resumo dos métodos de tingimento de ágatas é apresentado na Tabela 1.

Repara-se, no entanto, que, em vários processos aplicados, há uma mudança na coloração com o passar do tempo e esse fenômeno nunca foi estudado de forma sistemática. Assim, a implementação de medidas instrumentais para a medição de cor pode ser uma ferramenta para o controle de qualidade no setor. A colorimetria é uma técnica de avaliação de cor não destrutiva, onde é possível obter parâmetros de cor em poucos segundos. É uma técnica bem estabelecida na indústria de tintas e tem sido aplicada para controle de qualidade em diferentes situações no processamento de minérios, como em medidas de pureza de caulins (Varela et al., 2005), quantificação de pigmentos minerais (Tücks \& Beck, 2005) e na avaliação da qualidade de ocras (Elias et al., 2006).

Assim, o objetivo do presente trabalho foi aplicar a colorimetria como ferramenta para avaliar o comportamento

das cores das ágatas tingidas artificialmente. Estudou-se, também, a perda de cor dos principais tipos de coloração, considerando diferentes ambientes de exposição.

\section{Material e métodos}

Foram coletadas três chapas de ágatas de cada cor: procedimentos clássicos (verde, vermelho, azul e preto), tingimento orgânico (verde, vermelho, rosa e roxo), totalizando 24 amostras. As chapas apresentavam formato ovóide com diâmetro médio de $12 \mathrm{~cm}$ e espessura

Tabela 1 - Processos de tingimento de ágatas aplicados no Estado do Rio Grande do Sul.

\begin{tabular}{|c|c|c|}
\hline & Coloração & Processo \\
\hline \multirow{4}{*}{ 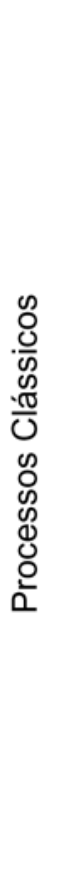 } & Verde & $\begin{array}{l}\text { Imersão das ágatas em uma solução } \\
\text { aquosa de ácido crômico e cloreto de } \\
\text { amônia. Após, procede-se à queima em } \\
\text { temperatura de } 150 \text { a } 300^{\circ} \mathrm{C} \text {. }\end{array}$ \\
\hline & Vermelha & $\begin{array}{l}\text { Imersão das ágatas em uma solução } \\
\text { aquosa de ácido nítrico, perclorato de ferro e } \\
\text { sucata de ferro. Após, procede-se à queima } \\
\text { em temperatura de } 150 \text { a } 240^{\circ} \mathrm{C} \text {. }\end{array}$ \\
\hline & Azul & $\begin{array}{l}\text { Imersão das ágatas em uma solução } \\
\text { aquosa contendo ferrocianeto de potássio. } \\
\text { Posteriormente, coloca-se em um banho de } \\
\text { ácido sulfúrico comercial fervente. }\end{array}$ \\
\hline & Preto & $\begin{array}{l}\text { Imersão das ágatas em uma calda aquecida } \\
\text { de açúcar. Após, coloca-se em um banho de } \\
\text { ácido sulfúrico comercial fervente. Depois, } \\
\text { levam-se as peças à mufla para queima em } \\
\text { temperaturas entre } 150 \text { a } 200^{\circ} \mathrm{C} \text {. }\end{array}$ \\
\hline \multirow{4}{*}{ 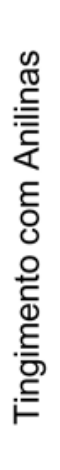 } & Verde & $\begin{array}{l}\text { Imersão das ágatas em uma solução } \\
\text { alcoólica do corante Verde Brilhante. }\end{array}$ \\
\hline & Vermelha & $\begin{array}{l}\text { Imersão das ágatas em uma solução } \\
\text { alcoólica de uma mistura dos corantes } \\
\text { Rodamina B e Laranja Básico. }\end{array}$ \\
\hline & Rosa & $\begin{array}{l}\text { Imersão das ágatas em uma solução } \\
\text { alcoólica do corante Rodamina B. }\end{array}$ \\
\hline & Roxo & $\begin{array}{l}\text { Imersão das ágatas em uma solução } \\
\text { alcoólica do corante Cristal Violeta. }\end{array}$ \\
\hline
\end{tabular}


média de $6 \mathrm{~mm}$. Foram divididas em três ambientes para exposição. No primeiro ambiente - escuro - havia total ausência de luz, sendo as chapas expostas à luminosidade somente durante as leituras do espectro de reflectância. O segundo grupo de chapas foi colocado em um local que representasse um ambiente com iluminação intermediária, simulando um ambiente doméstico. O terceiro conjunto de chapas sofreu as variações climáticas mais agressivas, pois as chapas foram colocadas ao ar livre, recebendo diretamente a ação da luz solar, de variações de temperatura e chuva.

Foram confeccionadas máscaras para fixar a chapa de ágata, com o intuito de manter a localização dos pontos analisados constantes, como ilustra a Figura 1. Cada amostra teve três pontos de leitura, que originaram valores médios. Procurou-se manter os locais de análises na mesma banda cristalográfica, para melhorar a reprodutibilidade dos resultados.

As leituras foram executadas com o auxílio de um espectrofotômetro MINOLTA CM-2600d, com esfera de integração associada a um filtro ultra-violeta. Foi usado como iluminante o $\mathrm{D}_{65}$, que representa a repartição espectral da luz do dia. A captação da reflectância simulou um observador a $10^{\circ}$. A calibração do aparelho foi executada no início do trabalho, tendo dois pontos de referência, o zero e o branco-padrão. A partir do espectro de reflectância da amostra, pode-se obter os parâmetros colorimétricos L*, a* e b* determinados pela CIE (Comission International de l'Eclairage) (CIE, 1986). Esses parâmetros representam os eixos de um diagrama tridimensional (Figura 2). Valores positivos de a* indicam cores vermelhas, enquanto que valores negativos representam cores verdes. Da mesma forma, valores positivos de $b^{*}$ demonstram cores amarelas e valores negativos demonstram cores azuis. $L^{*}$ é uma medida da escala de cinza, entre o preto e o branco, em uma faixa de medida que varia de $0-100$. O encontro dos três valores define uma cor.

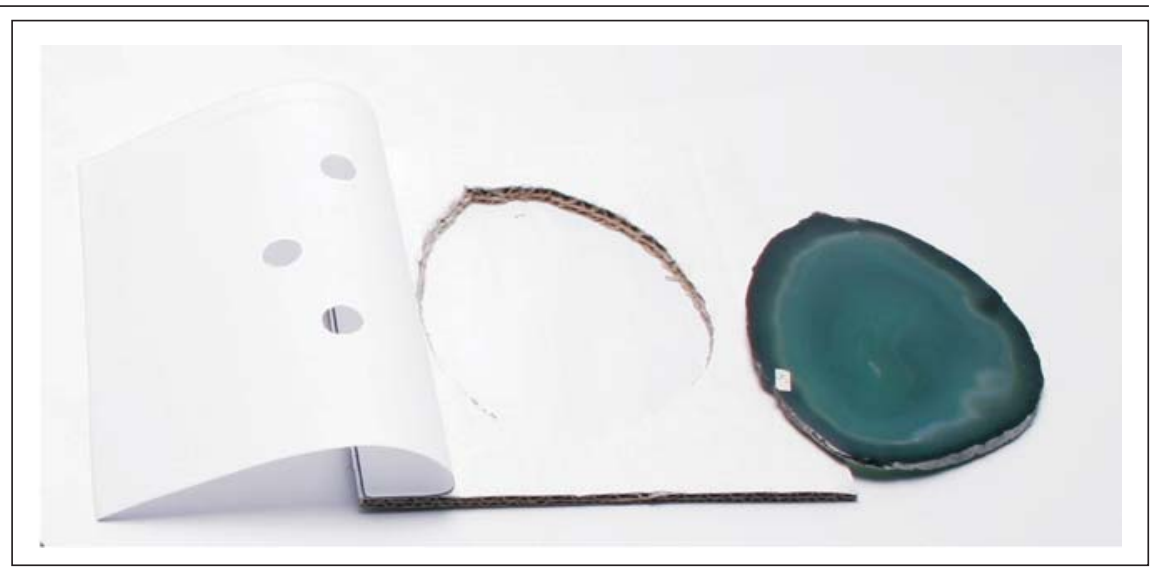

Figura 1 - Máscara fixadora da chapa de ágata.

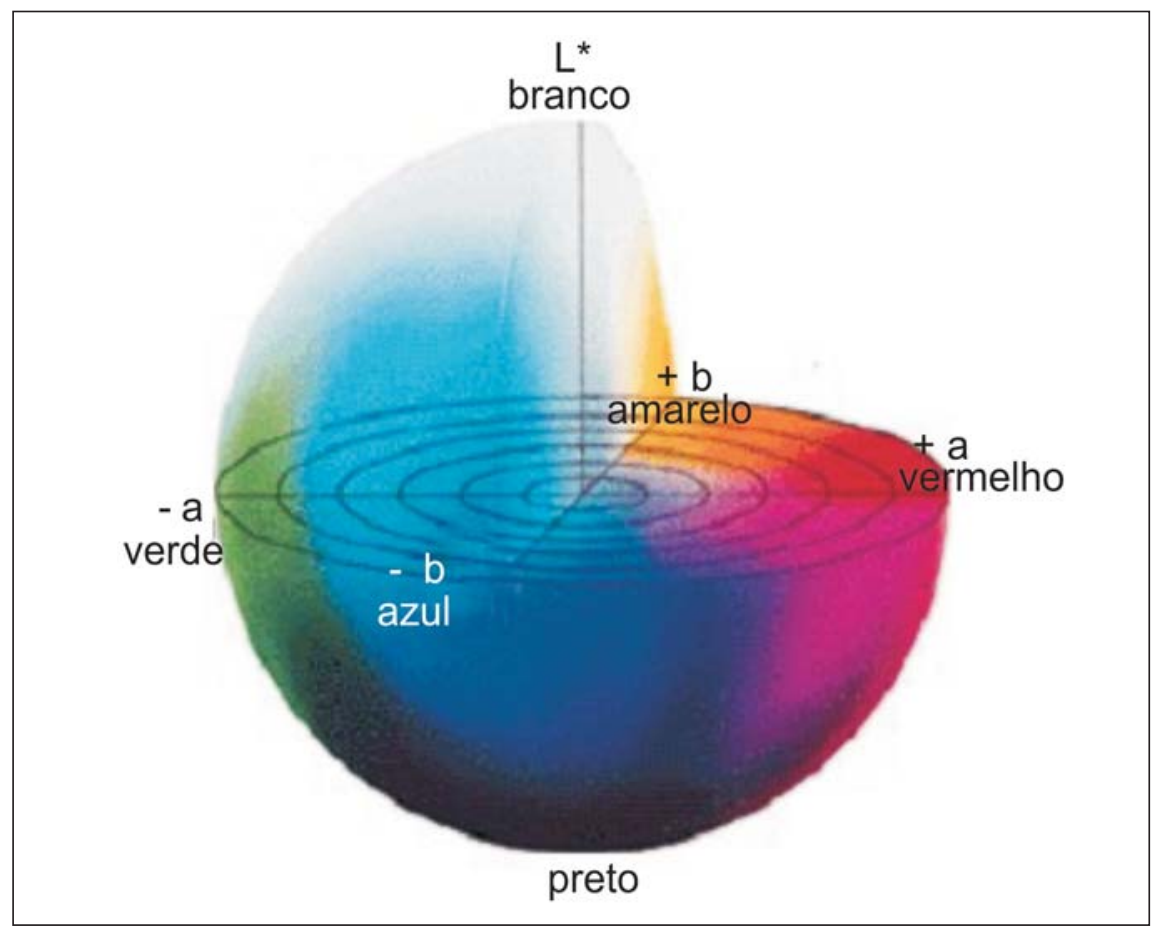

Figura 2 - Representação do sólido colorido para o espaço colorimétrico L*a*b*(Minolta, 1994).

Diferenças de cor $\left(\Delta \mathrm{E}^{*}{ }_{\mathrm{ab}}\right)$, que são importantes para avaliar relações visuais e numéricas (CIE, 1995), podem ser calculadas pela distâncias entre dois pontos no espaço tridimensional definido pelos parâmetros a*, b* e L*. Matematicamente, o parâmetro colorimétrico $\Delta \mathrm{E}$ é descrito pela Equação 1:

$$
\Delta \mathrm{E}_{\mathrm{ab}}^{*}=\sqrt{\left(\Delta L^{*}\right)^{2}+\left(\Delta a^{*}\right)^{2}+\left(\Delta b^{*}\right)^{2}}
$$

onde os valores de $\Delta \mathrm{L}^{*}, \Delta \mathrm{a}^{*}$ e $\Delta \mathrm{b}^{*}$ foram calculados pela diferença de valores medidos na primeira e última leitura nas 30 semanas de observação. A Tabela 2 mostra a classificação usada pela indústria de tintas em relação aos valores de $\Delta \mathrm{E}^{*}{ }_{\mathrm{ab}}$ para a percepção do olho humano. De modo geral, diferenças de cor, em duas amostras justapostas, podem ser distinguidas em valores de $\Delta \mathrm{E}^{*}{ }_{\mathrm{ab}}$ acima de 0,2-0,5 (DIN6174, 1979). 
Avaliação da perda da coloração artificial de ágatas

\section{Resultados e discussão}

A Tabela 3 apresenta os dados dos parâmetros colorimétricos obtidos na primeira leitura e após 30 semanas de exposição nos três ambientes avaliados (escuro, doméstico e ao ar livre).

Pode-se observar que os métodos clássicos de tingimento apresentam coloração estável. Perdas mínimas de cor foram observadas somente para a coloração azul e preta quando as ágatas com
Tabela 2 - Percepção humana para diferentes valores de $\Delta \mathrm{E}^{*}{ }_{\mathrm{ab}}$ empregada pela indústria de tintas (Norma DIN 6174, 1979).

\begin{tabular}{c|c}
\hline Diferenças $\left(\Delta \mathrm{E}^{*}{ }_{\mathrm{ab}}\right)$ & Classificação \\
\hline $0,0-0,2$ & Imperceptivel \\
\hline $0,2-0,5$ & Muito pequena \\
\hline $0,5-1,5$ & Pequena \\
\hline $1,5-3,0$ & Distinguivel \\
\hline $3,0-6,0$ & Facilmente distinguivel \\
\hline Maior que 6 & Muito grande \\
\hline
\end{tabular}

Tabela 3 - Parâmetros colorimétricos originais e após 30 semanas de exposição em diferentes ambientes para os distintos tipos de tingimento de ágatas.

\begin{tabular}{|c|c|c|c|c|c|c|c|c|c|c|}
\hline & & & \multicolumn{4}{|c|}{ Valores iniciais } & \multicolumn{4}{|c|}{ Valores após 30 semanas } \\
\hline & Cor & Ambientes & $L^{*}$ & $a^{*}$ & $b^{*}$ & $\Delta \mathrm{E}_{\mathrm{ab}}^{*}$ & $L^{*}$ & $a^{*}$ & $b^{*}$ & $\Delta \mathrm{E}_{\mathrm{ab}}^{*}$ \\
\hline \multirow{12}{*}{ 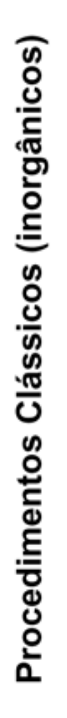 } & \multirow{3}{*}{$\frac{0}{\frac{0}{0}}$} & Escuro & 18,88 & $-21,65$ & 0,51 & 0,00 & 17,80 & $-20,31$ & 0,23 & 1,74 \\
\hline & & Doméstico & 27,85 & $-14,49$ & $-4,17$ & 0,00 & 29,25 & $-15,53$ & $-4,26$ & 1,74 \\
\hline & & Ao ar livre & 18,58 & $-19,21$ & 0,36 & 0,00 & 19,86 & $-18,21$ & 0,96 & 1,73 \\
\hline & \multirow{3}{*}{ 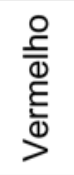 } & Escuro & 16,63 & 16,84 & 12,20 & 0,00 & 17,15 & 16,57 & 11,93 & 0,65 \\
\hline & & Doméstico & 16,02 & 18,92 & 14,70 & 0,00 & 21,78 & 18,67 & 16,29 & 5,98 \\
\hline & & Ao ar livre & 14,25 & 18,21 & 13,56 & 0,00 & 16,84 & 16,20 & 13,61 & 3,28 \\
\hline & \multirow{3}{*}{$\overline{\bar{N}}$} & Escuro & 3,96 & 1,83 & $-9,80$ & 0,00 & 4,38 & 1,79 & $-9,53$ & 0,49 \\
\hline & & Doméstico & 5,56 & 1,23 & $-11,77$ & 0,00 & 14,11 & 0,40 & $-18,10$ & 10,67 \\
\hline & & Ao ar livre & 11,36 & $-1,10$ & $-13,19$ & 0,00 & 35,71 & $-4,74$ & $-19,79$ & 25,49 \\
\hline & \multirow{3}{*}{$\begin{array}{l}\frac{0}{0} \\
\frac{0}{2}\end{array}$} & Escuro & 4,09 & 0,45 & $-0,27$ & 0,00 & 4,40 & 0,49 & $-0,22$ & 0,32 \\
\hline & & Doméstico & 3,20 & 1,12 & 0,45 & 0,00 & 6,02 & 1,39 & $-0,33$ & 2,93 \\
\hline & & Ao ar livre & 2,51 & 0,63 & 0,29 & 0,00 & 11,26 & 0,37 & $-3,90$ & 9,70 \\
\hline \multirow{12}{*}{ 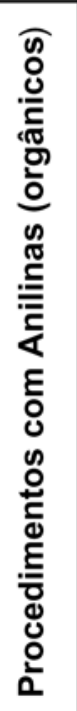 } & \multirow{3}{*}{$\frac{0}{\frac{0}{0}}$} & Escuro & 10,70 & $-14,03$ & $-15,18$ & 0,00 & 11,94 & $-14,55$ & $-15,31$ & 1,35 \\
\hline & & Doméstico & 15,60 & $-14,54$ & $-13,11$ & 0,00 & 35,38 & $-7,57$ & $-3,17$ & 23,21 \\
\hline & & Ao ar livre & 14,34 & $-14,38$ & $-17,20$ & 0,00 & 48,81 & 2,21 & 1,58 & 42,62 \\
\hline & \multirow{3}{*}{ 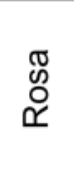 } & Escuro & 12,98 & 32,82 & $-13,24$ & 0,00 & 15,86 & 35,90 & $-12,54$ & 4,27 \\
\hline & & Doméstico & 11,53 & 31,94 & $-13,92$ & 0,00 & 40,06 & 14,10 & $-10,05$ & 33,86 \\
\hline & & Ao ar livre & 10,88 & 33,52 & $-13,02$ & 0,00 & 57,57 & $-0,02$ & $-3,99$ & 58,19 \\
\hline & \multirow{3}{*}{ 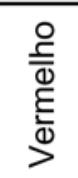 } & Escuro & 13,72 & 19,64 & $-1,93$ & 0,00 & 14,27 & 19,21 & $-1,19$ & 1,02 \\
\hline & & Doméstico & 3,70 & 7,60 & 0,61 & 0,00 & 12,17 & 13,27 & $-2,13$ & 10,56 \\
\hline & & Ao ar livre & 6,59 & 23,26 & 5,68 & 0,00 & 49,83 & 1,38 & $-8,92$ & 50,61 \\
\hline & \multirow{3}{*}{$\begin{array}{l}\stackrel{\text { }}{\not} \\
\stackrel{x}{\not ்}\end{array}$} & Escuro & 5,76 & 17,54 & $-22,99$ & 0,00 & 6,66 & 15,43 & $-20,96$ & 3,06 \\
\hline & & Doméstico & 4,74 & 12,96 & $-15,93$ & 0,00 & 19,54 & 9,82 & $-15,54$ & 15,13 \\
\hline & & Ao ar livre & 4,80 & 14,54 & $-21,31$ & 0,00 & 46,66 & $-1,62$ & $-9,09$ & 46,51 \\
\hline
\end{tabular}

480 REM: R. Esc. Minas, Ouro Preto, 60(3): 477-482, jul. set. 2007 
tais cores eram expostas ao ar livre. Porém, as ágatas tingidas com corantes orgânicos mostraram-se sensíveis à luz, apresentando perda de cor, tanto em ambiente doméstico, como ao ar livre.

Para exemplificar, as Figuras 3 e 4 mostram o deslocamento dos parâmetros colorimétricos no espaço $L^{*}$, a* e b* para o verde obtido com óxido de cromo e para o verde obtido com o corante Verde Brilhante. Pode-se observar que a cor obtida pelo método clássico está fixa no espaço colorimétrico, enquanto que a cor obtida pelo corante orgânico se desloca. O deslocamento se dá para cima e em direção ao centro do espaço tridimensional, o que demonstra que as pedras ornamentais ficam mais claras e com o tom acinzentado original.

Deve-se levar em consideração que a ágata em bruto apresenta tons que variam do cinza azulado para o cinza amarelado. Tubino (1998) obteve os seguintes índices colorimétricos para a ágata em bruto: L* entre 44,49 e 49,19; a* entre 3,38 e 4,56, b* entre 4,62 e 9,17.

Os resultados demonstram, também, o efeito da intensidade da luz. Quando mantidas em ambiente escuro, a cor se mantém parecida com a original por meses. Em ambiente doméstico, a perda de cor ocorre de forma gradual, mantendo-se ainda por meses, apesar de que com menor intensidade. Quando expostas à intempérie, a perda de cor pode ocorrer totalmente em poucas semanas. Observou-se, porém, que, em alguns casos, como no caso particular da peça tingida com o corante Verde Brilhante, a perda de cor pode salientar as diferenças entre as bandas cristalográficas, diminuindo o efeito borrado e aumentando a beleza do adorno.

O interesse por parte das empresas no tingimento com corantes orgânicos deve-se a fatores de ordem técnica e econômica. Os processos clássicos de tingimento são demorados (o tempo dos banhos podem chegar a semanas) e a quebra das peças no processo de queima pode acarretar em perdas de 30-40\%. Há, também, um alto custo envolvido no tratamento dos efluentes, que, dependendo do banho, podem conter alta aci-

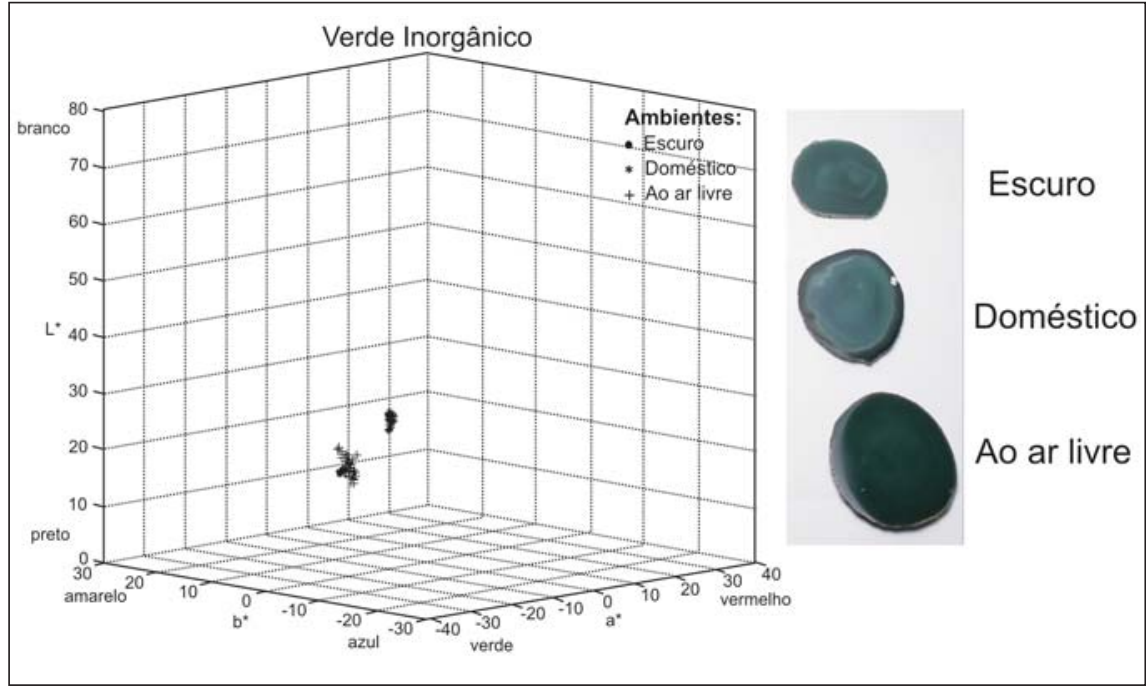

Figura 3 - Diagrama da evolução dos parâmetros colorimétricos $L^{*}$, $a^{*}$ e b*. Fotografia do estado final das chapas de ágatas tingidas com óxido de cromo.

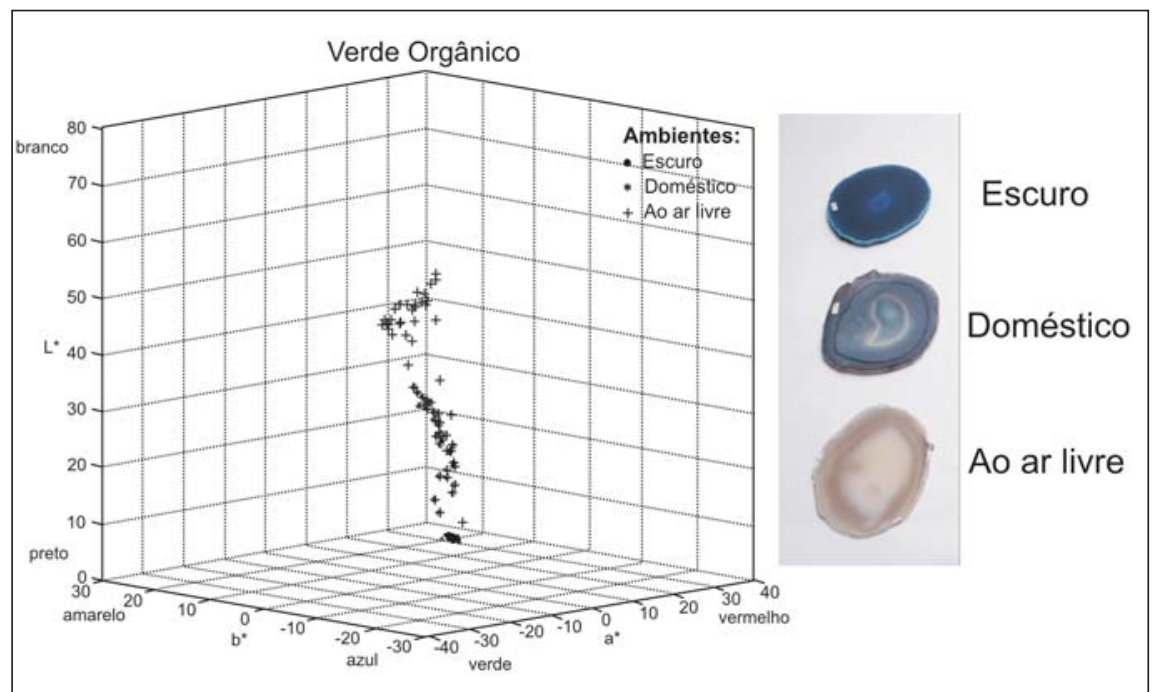

Figura 4 - Diagrama da evolução dos parâmetros colorimétricos $L^{*}, a^{*} e b^{*}$. Fotografia do estado final das chapas de ágatas tingidas com corante Verde Brilhante.

dez, Cr(VI), outros metais e cianetos. A coloração das ágatas com o uso dos corantes orgânicos permite a obtenção de cores diferentes dos processos clássicos (como o rosa e o roxo), o tempo de produção é de poucos dias, não é necessária uma etapa de aquecimento das chapas para a fixação da cor e o tratamento do efluente pode ser realizado de forma simplificada com o uso de hipoclorito de sódio (Pizzolato et al., 2002).

Deve-se ressaltar, no entanto, que a perda de cor passa despercebida pela maior parte dos clientes, apesar dos valores de $\Delta \mathrm{E}^{*}{ }_{\mathrm{ab}}$ registrados serem percep- tíveis pelo olho humano (Tabela 2). Isso se deve ao fato de que a perda ocorre de forma gradual e que o consumidor não tem como forma de comparação a cor original da pedra ornamental quando adquirida. Nesse sentido, a colorimetria diferencial pode ser uma ferramenta útil no estabelecimento de padrões de qualidade para a coloração de ágatas.

Por fim, sugere-se que o consumidor deva ser informado sobre o tipo de tingimento empregado e quais as recomendações devam ser seguidas para que se evite, ou pelo menos minimize, a perda na coloração. 


\section{Conclusões}

O sistema colorimétrico CIE $\mathrm{L} * \mathrm{a} * \mathrm{~b} *$ mostrou-se capaz de avaliar as modificações na cor em ágatas coloridas artificialmente. Os métodos clássicos de tingimento proporcionam cores permanentes, enquanto que o tingimento com corantes orgânicos produz cores passíveis de descoloração. Dependendo do grau de luminosidade e tempo de exposição, a perda de cor pode ser praticamente total.

\section{Agradecimentos}

Os autores agradecem ao CNPq e à Fapergs pelo auxílio financeiro para o desenvolvimento do presente trabalho.

\section{Referências bibliográficas}

CIE. Comission Internationale de L'éclairage. Technical Report. Viena, Austria: CIE 15.2-1986.
CIE. Comission Internationale de L’éclairage. Industrial Colour-Difference Evaluation. Viena, Austria: CIE 116-1995.

DIN. DEUTSCHE INSTITUT für NORMUNG. DIN 6174. Farbmetrische Bestimmung von Farbabständen Bei Körperfarben nach der CIELAB-Formel. Berlim und Köln: Beuth Verlag, 1979.

DNPM. Ágata do Rio Grande do Sul. Brasília, 1998. (Série Difusão Tecnológica).

ELIAS, M., CHARTIER, C., PRÉVOT, G., GARAY, H., VIGNAUD, C. The colour of ochres explained by their composition. Materials Science and Engineering B, 127, 7080, 2006.

KNECHT,T. Coloração artificial de ágatas. Revista da Associação Brasileira de Gemologia, v.7, p.1-9, 1957.

MINOLTA. Precise color communications. Color Control from Feeling to Instrumentation. Osaka, Japan: 1994.

PIZZOLATO, T.M., CARISSIMI, E., MACHADO, E.L. SCHNEIDER, I.A.H. Colour removal with $\mathrm{NaClO}$ of dye wastewater from an agate-processing plant in Rio Grande do Sul. International Journal of Mineral Processing, v.65, p.203-211, 2002.

SAMPAIO, C.H., TUBINO, L.C.B., Estudo morfológico da ágata em bruto e sua influência no processo de tingimento industrial em bruto. Acta Geológica Leopoldense, v.20, n.8, p.43-48, 1999.

TUBINO, L.C. Tratamento industrial da ágata em bruto no Estado do Rio Grande do Sul. Porto Alegre: PPGEM, UFRGS, 1998. (Dissertação de Mestrado).

TÜCKS, A., BECK, H.P. The photochromic effect of bismuth vanadate pigments. Part I: Synthesis, characterization and lightfastness of pigment coatings. Journal of Solid State Chemistry, v.178, p. 1145-1156, 2005.

VARELA, J., PEIXOTO, C.A., GLIESE, R., PETTER, C.O. Controle de qualidade no processamento de polpas de caulim utilizando propriedades ópticas. Rem - Revista Escola de Minas, v.58, n.3, p.201-206, 2005.

Artigo recebido em 05/08/2006 e aprovado em 16/02/2007.

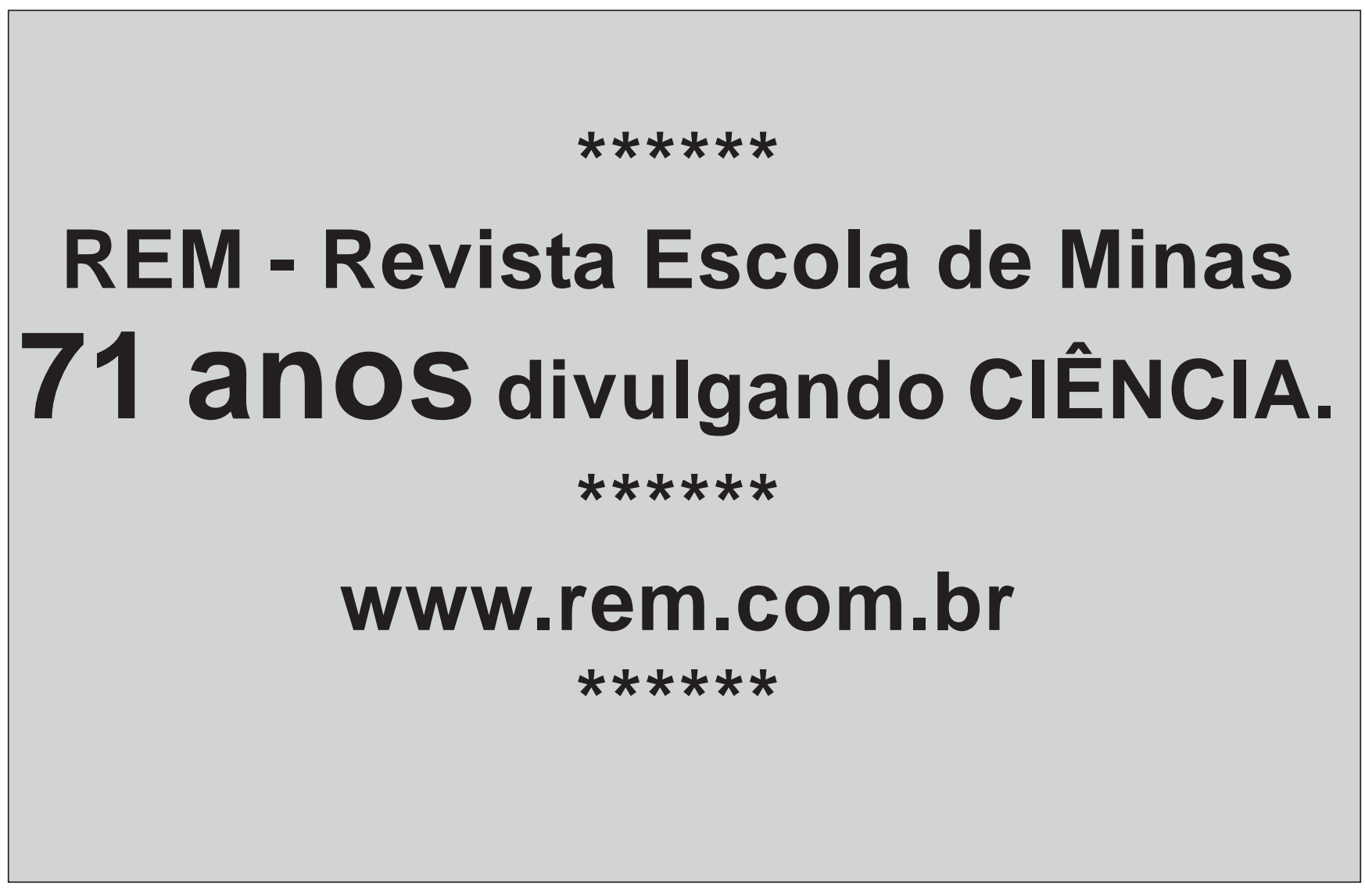

